\title{
Symmetries for generating string cosmologies *
}

\author{
E. J. Copeland ${ }^{1}$, James E. Lidsey ${ }^{1,2,3}$ and David Wands ${ }^{4}$ \\ ${ }^{1}$ Centre for Theoretical Physics, University of Sussex, Brighton, BN1 9QH, U.K. \\ ${ }^{2}$ Astronomy Centre, University of Sussex, Brighton, BN1 9QH, U.K. \\ ${ }^{3}$ Astronomy Unit, School of Mathematical Sciences, Queen Mary and Westfield, Mile End Road, London, E1 4NS, U.K. \\ ${ }^{4}$ School of Computer Science and Mathematics, University of Portsmouth, Portsmouth, PO1 2EG, U.K.
}

(July 5, 2018)

\begin{abstract}
We discuss the symmetry properties of the low-energy effective action of the type IIB superstring that may be employed to derive four-dimensional solutions. A truncated effective action, compactified on a six-torus, but including both Neveu/Schwarz-Neveu/Schwarz and Ramond-Ramond field strengths, can be expressed as a non-linear sigma model which is invariant under global $\operatorname{SL}(3, \mathrm{R})$ transformations. This group contains as a sub-group the $\mathrm{SL}(2, \mathrm{R})$ symmetry of the ten-dimensional theory and a discrete $\mathrm{Z}_{2}$ reflection symmetry which leads to a further $\mathrm{SL}(2, \mathrm{R})$ sub-group. The symmetries are employed to analyse a general class of spatially homogeneous cosmological solutions with non-trivial Ramond-Ramond fields.
\end{abstract}

PACS numbers: 98.80.Cq, 11.25.-w, 04.50.+h

\section{INTRODUCTION}

String theory has entered a new era. There is now strong evidence that the five separate perturbative string theories are related non-perturbatively by duality symmetries [1 5 . 5 . This suggests that the five theories may have a common origin in a more fundamental theory (M-theory), the low-energy limit of which is eleven-dimensional (11-D), $N=1$ supergravity. The symmetries of the type II string theories in particular have a number of interesting features [3]. The equations of motion of $10-\mathrm{D}$ type IIB supergravity are symmetric under global $\mathrm{SL}(2, \mathrm{R})$ transformations. The subgroup $\mathrm{SL}(2, \mathrm{Z})$ is the conjectured S-duality of the type IIB superstring [3]. This relates the strong- and weakcoupling regimes of the theory and interchanges Neveu/Schwarz-Neveu/Schwarz (NS-NS) and Ramond-Ramond (RR) charges.

Type IIA and IIB supergravity theories are equivalent after toroidal compactification [6]. Compactification of the low-energy effective type IIB action on a six-torus results in $N=8$ supergravity, which may also be derived by toroidally compactifying $11-\mathrm{D}, N=1$ supergravity [7]. This contains seventy scalar and pseudo-scalar fields that parametrize the $\mathrm{E}_{7(7)} /\left[\mathrm{SU}(8) / \mathrm{Z}_{2}\right]$ coset and the field equations are invariant under the global action of the group $\mathrm{E}_{7(7)}$. The discrete subgroup $\mathrm{E}_{7(7)}(\mathrm{Z})$ is the conjectured U-duality of the type II superstring and contains the T-duality group $\mathrm{O}(6,6 ; \mathrm{Z})$ and $\mathrm{S}-$ duality group $\mathrm{SL}(2, \mathrm{Z})[3]$.

The purpose of the present paper is to consider a truncated sector of $4-\mathrm{D}, N=8$ supergravity. We derive a $4-\mathrm{D}$ low-energy effective action that includes non-trivial interaction terms between the NS-NS and RR form fields, but maintains enough simplicity that exact solutions to the field equations can be found analytically and analysed in detail. A study of the symmetries of such an effective action is important because these provide a powerful method for generating new string solutions from existing vacuum solutions. It is known that there exists a global $\mathrm{O}(d, d)$ symmetry in the field equations when a string background admits $d$ Abelian isometries 86,9]. (For an extensive review, see, e.g., Ref. [2]). For example, this symmetry has been employed to generate curved backgrounds with a time-dependent tensor potential from flat solutions [10] and homogeneous cosmologies [11. On the other hand the presence of an SL(2,R) symmetry [12] in the NS-NS sector of the theory can been used to generate a time-dependent field-strength [13 16]. Such 4-D solutions derived from the low-energy effective action are interesting because they represent a first approximation to string backgrounds that are exact to all orders. Furthermore, the possible role played by RR fields in cosmology may be investigated using such an action.

In view of this, we compactify the low-energy effective type IIB theory on an isotropic six-torus and only include the variation of the moduli and form-fields on the external 4-D spacetime. In four dimensions, the three-form field strengths are dual to one-forms and this correspondence is employed to derive a dual action in terms of five scalar and pseudo-scalar 'axion' fields. We find that these fields parametrize an $\mathrm{SL}(3, \mathrm{R}) / \mathrm{SO}(3)$ coset. The global $\mathrm{SL}(2, \mathrm{R})$

*To appear in Physical Review D. Copyright 1998 by The American Physical Society 
symmetry of the 10-D supergravity action is preserved in 4-D as a subgroup of the SL(3,R) symmetry. We also identify a discrete $\mathrm{Z}_{2}$ symmetry, corresponding to a subgroup of the full $\mathrm{O}(6,6 ; \mathrm{Z}) \mathrm{T}$-duality, which leads to a further $\mathrm{SL}(2, \mathrm{R})$ symmetry that may be viewed as a 'mirror' image of the original SL(2,R) symmetry.

The paper is organised as follows. We derive the dual effective action in Section II and discuss its global symmetries in Section III. In Section IV, we present a general class of homogeneous cosmological solutions as an example of how 4-D spacetimes with non-trivial NS-NS and RR fields may be derived by employing the symmetries of the action. Cosmological solutions obtained by employing the full $\mathrm{SL}(3, \mathrm{R})$ symmetry [17] generalise results previously obtained from a single $\mathrm{SL}(2, \mathrm{R})$ transformation acting on the NS-NS solutions 18,19 .

\section{THE EFFECTIVE ACTION}

The low-energy limit of the type IIB superstring is $10-\mathrm{D}, N=2$ chiral supergravity [20]. The bosonic massless excitations in the NS-NS sector are the dilaton, $\Phi$, the metric, $g_{M N}$, and the antisymmetric, two-form potential, $B_{M N}^{(1)}$. The RR sector contains a scalar axion field, $\chi$, a two-form potential, $B_{M N}^{(2)}$, and a four-form potential, $D_{M N P Q}$. The equation of motion for the four-form cannot be derived from a covariant 10-D action and we therefore assume that this field vanishes 21]. The field equations for the remaining degrees of freedom can be derived by extremizing the action [22]:

$$
S_{\mathrm{IIB}}=\int d^{10} x \sqrt{-g_{10}}\left\{e^{-\Phi}\left[R_{10}+(\nabla \Phi)^{2}-\frac{1}{12}\left(H^{(1)}\right)^{2}\right]-\frac{1}{2}(\nabla \chi)^{2}-\frac{1}{12}\left(H^{(1)} \chi+H^{(2)}\right)^{2}\right\},
$$

where $R_{10}$ is the Ricci curvature scalar, $g_{10} \equiv \operatorname{det}\left(g_{M N}\right)$ and

$$
H_{M N P}^{(i)} \equiv \partial_{[M} B_{N P]}^{(i)}
$$

are the field strengths of the two-form potentials $B_{M N}^{(i)}$. The RR terms do not couple directly to the $10-\mathrm{D}$ dilaton in the string frame, and the dilaton field is minimally coupled to the metric in the conformally related, 10-D Einstein frame:

$$
\hat{g}_{M N}=e^{-\Phi / 4} g_{M N}
$$

We then have

$$
S_{\text {IIB }}=\int d^{10} x \sqrt{-\hat{g}_{10}}\left\{\hat{R}_{10}-\frac{1}{8}(\hat{\nabla} \Phi)^{2}-\frac{1}{2} e^{\Phi}(\hat{\nabla} \chi)^{2}-\frac{1}{12} e^{\Phi / 2}\left(\hat{H}^{(1)}\right)^{2}-\frac{1}{12} e^{-\Phi / 2}\left(\hat{H}^{(1)} \chi+\hat{H}^{(2)}\right)^{2}\right\}
$$

and Eq. 2.4) can be written as [3,23

$$
S_{\text {IIB }}=\int d^{10} x \sqrt{-\hat{g}_{10}}\left\{\hat{R}_{10}+\frac{1}{4} \operatorname{Tr}\left(\hat{\nabla} M \hat{\nabla} M^{-1}\right)-\frac{1}{12} \hat{H}^{T} M \hat{H}\right\}
$$

where

$$
M \equiv\left(\begin{array}{cc}
e^{\Phi / 2} & \chi e^{\Phi / 2} \\
\chi e^{\Phi / 2} & e^{-\Phi / 2}+\chi^{2} e^{\Phi / 2}
\end{array}\right)
$$

and

$$
\hat{H}=\left(\begin{array}{c}
\hat{H}^{(2)} \\
\hat{H}^{(1)}
\end{array}\right)
$$

It follows that $M^{T} J M=J$, where

\footnotetext{
${ }^{1}$ In this paper, Upper case Latin indices take values in the range $A=(0,1, \ldots, 9)$, lower case Latin indices vary from $a=(4,5, \ldots 9)$ and Greek indices are given by $\mu=(0,1,2,3)$. The signature of spacetime is $(-,+,+, \ldots,+)$.
} 


$$
J \equiv\left(\begin{array}{cc}
0 & 1 \\
-1 & 0
\end{array}\right), \quad J^{2}=-I
$$

is the $\mathrm{SL}(2, \mathrm{R})$ metric. Thus, $M$ is a member of the group $\mathrm{SL}(2, \mathrm{R})$ and the action $(2.5)$ is invariant under the global $\mathrm{SL}(2, \mathrm{R})$ transformation [3, 23]:

$$
\bar{M}=\Sigma M \Sigma^{T}, \quad \overline{\hat{g}}_{M N}=\hat{g}_{M N}, \quad \overline{\hat{H}}=\left(\Sigma^{T}\right)^{-1} \hat{H},
$$

where

$$
\Sigma \equiv\left(\begin{array}{cc}
D & C \\
B & A
\end{array}\right), \quad A D-B C=1 .
$$

The invariance of the 10-D Einstein metric $\hat{g}_{M N}$ implies that the string metric $g_{M N}$ transforms under Eq. (2.9) as

$$
\bar{g}_{M N} e^{-\bar{\Phi} / 4}=g_{M N} e^{-\Phi / 4} .
$$

The four-form transforms as a singlet.

\section{A. Reduced 4-D action}

The toroidal compactification of the type IIB theory (2.1) has recently been discussed 22,24 27. Maharana 24. and Roy [25] compactified down to $D$ dimensions and showed how the $\mathrm{SL}(2, \mathrm{R})$ symmetry discussed above is respected in lower dimensions. We compactify to four dimensions with the simplest toroidal ansatz:

$$
d s_{10}^{2}=g_{\mu \nu}(x) d x^{\mu} d x^{\nu}+e^{y(x) / \sqrt{3}} \delta_{a b} d X^{a} d X^{b},
$$

where $y$ is a modulus field describing the volume of the internal space. This field represents the 'breathing mode' of the internal space. We assume that all fields are independent of the internal coordinates $X^{a}$. We do not consider the vector fields arising from the metric components or the moduli originating from the compactification of the threeforms. Although this is a more severe truncation of the toroidally compactified action than the one considered by Maharana [24] and Roy [25], it has the advantage that the interactions between the NS-NS and RR fields can be quantitatively analysed. Each form field is reduced to a single degree of freedom and this allows valuable insight to be gained into the role played by the RR sector.

The reduced $4-\mathrm{D}$ effective action in the string frame is given by

$$
\begin{gathered}
S_{4}=\int d^{4} x \sqrt{-g}\left\{e^{-\varphi}\left[R+(\nabla \varphi)^{2}-\frac{1}{2}(\nabla y)^{2}-\frac{1}{12}\left(H^{(1)}\right)^{2}\right]\right. \\
\left.-\frac{1}{2} e^{\sqrt{3} y}(\nabla \chi)^{2}-\frac{1}{12} e^{\sqrt{3} y}\left(H^{(1)} \chi+H^{(2)}\right)^{2}\right\},
\end{gathered}
$$

where

$$
\varphi \equiv \Phi-\sqrt{3} y
$$

represents the effective dilaton in the 4-D spacetime. The corresponding action in the 4-D Einstein frame

$$
\tilde{g}_{\mu \nu}=e^{-\varphi} g_{\mu \nu}
$$

is then given by

$$
\begin{array}{r}
S_{4}=\int d^{4} x \sqrt{-\tilde{g}}\left\{\tilde{R}_{4}-\frac{1}{2}(\tilde{\nabla} \varphi)^{2}-\frac{1}{2}(\tilde{\nabla} y)^{2}-\frac{1}{12} e^{-2 \varphi}\left(\tilde{H}^{(1)}\right)^{2}\right. \\
\left.-\frac{1}{2} e^{\sqrt{3} y+\varphi}(\tilde{\nabla} \chi)^{2}-\frac{1}{12} e^{\sqrt{3} y-\varphi}\left(\tilde{H}^{(1)} \chi+\tilde{H}^{(2)}\right)^{2}\right\} .
\end{array}
$$

Note that the 4-D Einstein frame metric, Eq. (2.15), differs from the corresponding part of the 10-D Einstein frame metric (2.3) by the conformal factor

$$
\Omega^{2}=e^{\Phi / 4-\varphi}=e^{\sqrt{3}(y-\sqrt{3} \varphi) / 4} .
$$




\section{B. The Dual Action}

In what follows we shall always refer to the metric in the 4-D Einstein frame and so we drop tildes for notational simplicity. The field equations for the three-forms in the 4-D Einstein frame are given by

$$
\begin{aligned}
\nabla_{\mu}\left[e^{\sqrt{3} y-\varphi}\left(\chi H^{(1) \mu \nu \lambda}+H^{(2) \mu \nu \lambda}\right)\right] & =0 \\
\nabla_{\mu}\left[e^{-2 \varphi} H^{(1) \mu \nu \lambda}+\chi e^{\sqrt{3} y-\varphi}\left(\chi H^{(1) \mu \nu \lambda}+H^{(2) \mu \nu \lambda}\right)\right] & =0 .
\end{aligned}
$$

In four dimensions the three-form field strengths are dual to one-forms:

$$
\begin{gathered}
H_{\mu \nu \lambda}^{(1)} \equiv \epsilon_{\mu \nu \lambda \kappa} K^{\kappa} \\
H_{\mu \nu \lambda}^{(2)} \equiv \epsilon_{\mu \nu \lambda \kappa} J^{\kappa},
\end{gathered}
$$

where $\epsilon_{\mu \nu \lambda \kappa}$ is the covariantly constant four-form. The field equations (2.18) and (2.19) take the form

$$
\begin{aligned}
\epsilon^{\mu \nu \lambda \kappa} \nabla_{\mu}\left[e^{\sqrt{3} y-\varphi}\left(\chi K_{\kappa}+J_{\kappa}\right)\right] & =0 \\
\epsilon^{\mu \nu \lambda \kappa} \nabla_{\mu}\left[e^{-2 \varphi} K_{\kappa}+\chi e^{\sqrt{3} y-\varphi}\left(\chi K_{\kappa}+J_{\kappa}\right)\right] & =0
\end{aligned}
$$

when written in terms of the dual one-forms.

These dual forms may be written in terms of the gradients of two pseudo-scalar 'axion' fields. Equation (2.22) requires that

$$
e^{\sqrt{3} y-\varphi}\left(\chi K_{\kappa}+J_{\kappa}\right)=\nabla_{\kappa} \sigma_{2},
$$

where $\sigma_{2}$ is any scalar function. Substituting this into Eq. (2.23) implies that

$$
e^{-2 \varphi} K_{\kappa}+\chi \nabla_{\kappa} \sigma_{2}=\nabla_{\kappa} \sigma_{1}
$$

where $\sigma_{1}$ is a second arbitrary scalar function. These together imply that the field equations (2.18) and (2.19) are automatically satisfied by

$$
\begin{aligned}
& H^{(1) \mu \nu \lambda}=\epsilon^{\mu \nu \lambda \kappa} e^{2 \varphi}\left(\nabla_{\kappa} \sigma_{1}-\chi \nabla_{\kappa} \sigma_{2}\right) \\
& H^{(2) \mu \nu \lambda}=\epsilon^{\mu \nu \lambda \kappa}\left[e^{\varphi-\sqrt{3} y} \nabla_{\kappa} \sigma_{2}-\chi e^{2 \varphi}\left(\nabla_{\kappa} \sigma_{1}-\chi \nabla_{\kappa} \sigma_{2}\right)\right] .
\end{aligned}
$$

It should be emphasised that the definitions of both scalar fields $\sigma_{i}$ are arbitrary up to a redefinition $\sigma_{i} \rightarrow \sigma_{i}+f_{i}$, where $f_{i}$ represents an arbitrary scalar function. In this sense, therefore, there is no unique definition of the pseudoscalar axion fields. However, when $H_{\mu \nu \lambda}^{(2)}=\chi=0$, we see that our definition of $\sigma_{1}$ coincides with the usual definition of the axion that is dual to the NS-NS three-form field strength [12, 13, 28, 15 .

Although the field equations (2.18) and (2.19) for the three-forms are now automatically satisfied by the dual ansatz, we must also impose the Bianchi identities

$$
\nabla_{[\mu} H_{\nu \lambda \kappa]}^{(i)} \equiv 0
$$

that arise because the three-form field strengths are defined in terms of the gradients of two-form potentials. These correspond to the constraint equations

$$
\begin{aligned}
\nabla_{\rho}\left[e^{2 \varphi}\left(\nabla^{\rho} \sigma_{1}-\chi \nabla^{\rho} \sigma_{2}\right)\right] & =0 \\
\nabla_{\rho}\left[e^{-\sqrt{3} y+\varphi} \nabla^{\rho} \sigma_{2}-\chi e^{2 \varphi}\left(\nabla^{\rho} \sigma_{1}-\chi \nabla^{\rho} \sigma_{2}\right)\right] & =0
\end{aligned}
$$

on the fields $\sigma_{i}$. They are interpreted in the dual ansatz as field equations that can be derived from the effective action

$$
\begin{gathered}
S_{4 *}=\int d^{4} x \sqrt{-g}\left[R-\frac{1}{2}(\nabla \varphi)^{2}-\frac{1}{2}(\nabla y)^{2}-\frac{1}{2} e^{\sqrt{3} y+\varphi}(\nabla \chi)^{2}\right. \\
\left.-\frac{1}{2} e^{-\sqrt{3} y+\varphi}\left(\nabla \sigma_{2}\right)^{2}-\frac{1}{2} e^{2 \varphi}\left(\nabla \sigma_{1}-\chi \nabla \sigma_{2}\right)^{2}\right] .
\end{gathered}
$$


This action is not identical to the original action (2.16) because the roles of the Bianchi identities and the field equations are interchanged. Nevertheless, the two descriptions are dynamically equivalent as long as the field equations are satisfied, and it should be emphasised that either form of the action should only be viewed as an effective action which reproduces the correct equations of motion. With this in mind, we refer to this dual action when investigating the symmetries of the $4-\mathrm{D}$ theory. The equations of motion for the five scalar fields are then given by Eqs. (2.29) and 2.30 , together with

$$
\begin{array}{r}
\square \varphi=\frac{1}{2} e^{\sqrt{3} y+\varphi}(\nabla \chi)^{2}+\frac{1}{2} e^{-\sqrt{3} y+\varphi}\left(\nabla \sigma_{2}\right)^{2}+e^{2 \varphi}\left(\nabla \sigma_{1}-\chi \nabla \sigma_{2}\right)^{2} \\
\square y=\frac{\sqrt{3}}{2} e^{\sqrt{3} y+\varphi}(\nabla \chi)^{2}-\frac{\sqrt{3}}{2} e^{-\sqrt{3} y+\varphi}\left(\nabla \sigma_{2}\right)^{2} \\
\nabla^{\mu}\left(e^{\sqrt{3} y+\varphi} \nabla_{\mu} \chi\right)=-e^{2 \varphi} \nabla^{\mu} \sigma_{2}\left(\nabla_{\mu} \sigma_{1}-\chi \nabla_{\mu} \sigma_{2}\right) .
\end{array}
$$

\section{Conserved currents}

The one-forms $K$ and $J$ defined in Eqs. 2.20) and (2.21) can be written in terms of the pseudo-scalar axion fields using Eqs. (2.24) and (2.25). This yields

$$
\begin{gathered}
K_{\mu}=e^{2 \varphi}\left(\nabla_{\mu} \sigma_{1}-\chi \nabla_{\mu} \sigma_{2}\right) \\
J_{\mu}=e^{-\sqrt{3} y+\varphi} \nabla_{\mu} \sigma_{2}-\chi K_{\mu}
\end{gathered}
$$

and the Bianchi identities (2.29) and (2.30) simply correspond to the requirement that these currents are conserved:

$$
\nabla^{\mu} K_{\mu}=0, \quad \nabla^{\mu} J_{\mu}=0 .
$$

In terms of the original three-form field strengths, these currents are topologically conserved due to the Bianchi identities, but in the dual formulation they are Noether currents conserved due to the symmetry of the action (2.31).

The conserved currents allow us to integrate out the kinetic terms for the pseudo-scalar axion fields $\sigma_{i}$. This reduces the field equations 2.32 2.34 to

$$
\begin{array}{r}
\square \varphi=\frac{1}{2} e^{\sqrt{3} y+\varphi}(\nabla \chi)^{2}-\frac{\partial V}{\partial \varphi} \\
\square y=\frac{\sqrt{3}}{2} e^{\sqrt{3} y+\varphi}(\nabla \chi)^{2}-\frac{\partial V}{\partial y} \\
\nabla^{\mu}\left(e^{\sqrt{3} y+\varphi} \nabla_{\mu} \chi\right)=-\frac{\partial V}{\partial \chi},
\end{array}
$$

where the effective interaction potential, $V$, for the fields $\varphi, y$ and $\chi$ is given by

$$
V=-\frac{1}{2} g^{\mu \nu}\left[e^{\sqrt{3} y-\varphi}\left(J_{\mu}+\chi K_{\mu}\right)\left(J_{\nu}+\chi K_{\nu}\right)+e^{-2 \varphi} K_{\mu} K_{\nu}\right]
$$

This arises due to the three-form field strengths and may be expressed in a SL(2,R) invariant form, as will be shown in the following section.

Note that the field equation for the field $\chi$, Eq. (2.34), can also be written as

$$
\nabla^{\mu}\left(e^{\sqrt{3} y+\varphi} \nabla_{\mu} \chi\right)=-K_{\mu} \nabla^{\mu} \sigma_{2}
$$

Since $\nabla^{\mu} K_{\mu}=0$, we deduce that

$$
L_{\mu}=e^{\sqrt{3} y+\varphi} \nabla_{\mu} \chi+\sigma_{2} K_{\mu}
$$

where $\nabla^{\mu} L_{\mu}=0$. Thus, $L_{\mu}$ is the third conserved current for the form-fields, independent of $K_{\mu}$ and $J_{\mu}$. This does not allow us to simplify the equations (2.38 2.40) any further as we simply swap our ignorance of $\nabla \chi$ for our ignorance of $\sigma_{2}$. However, it is indicative of a further symmetry of the dual action which we discuss in the following section. 


\section{SYMMETRIES OF THE DUAL ACTION}

\section{A. $\mathrm{SL}(3, \mathbf{R})$ symmetry}

The effective action (2.31) can be expressed as a 4-D, non-linear sigma-model:

$$
S_{4 *}=\int d^{4} x \sqrt{-g}\left[R-\mathcal{G}_{A B}(\varphi) \nabla \varphi^{A} \nabla \varphi^{B}\right]
$$

where the scalar fields $\varphi^{A}=\left(\varphi, y, \chi, \sigma_{1}, \sigma_{2}\right),(A, B)=(1,2, \ldots, 5)$ may be viewed as coordinates on the target space with metric

$$
\mathcal{G}=\mathcal{G}_{A B} d \varphi^{A} d \varphi^{B}=\frac{1}{2} d \varphi^{2}+\frac{1}{2} d y^{2}+\frac{1}{2} e^{2 \varphi}\left(d \sigma_{1}-\chi d \sigma_{2}\right)^{2}+\frac{1}{2} e^{\varphi}\left[e^{\sqrt{3} y} d \chi^{2}+e^{-\sqrt{3} y} d \sigma_{2}^{2}\right] .
$$

Eq. (3.2) is formally identical to the target space considered by Gal'tsov, Garcia and Kechkin within the context of 5-D, Kaluza-Klein theory admitting two commuting Killing vectors [29]. Maison first showed that this target space represents the $\mathrm{SL}(3, \mathrm{R}) / \mathrm{SO}(3)$ coset corresponding to a homogeneous symmetric Riemannian space, where the group $\mathrm{SL}(3, \mathrm{R})$ acts transitively [30]. It can be shown by employing the Gauss decomposition of the general SL(3,R) matrix that the action (2.31) may be written in the form 29.

$$
S_{4 *}=\int d^{4} x \sqrt{-g}\left[R+\frac{1}{4} \operatorname{Tr}\left[\nabla U \nabla U^{-1}\right]\right],
$$

where

$$
U \equiv e^{\varphi+y / \sqrt{3}}\left(\begin{array}{ccc}
1 & \chi & \sigma_{1}-\chi \sigma_{2} \\
\chi & \chi^{2}+e^{-\varphi-\sqrt{3} y} & \chi\left(\sigma_{1}-\chi \sigma_{2}\right)-\sigma_{2} e^{-\varphi-\sqrt{3} y} \\
\sigma_{1}-\chi \sigma_{2} & \chi\left(\sigma_{1}-\chi \sigma_{2}\right)-\sigma_{2} e^{-\varphi-\sqrt{3} y} & \left(\sigma_{1}-\chi \sigma_{2}\right)^{2}+\sigma_{2}^{2} e^{-\varphi-\sqrt{3} y}+e^{-2 \varphi}
\end{array}\right)
$$

is a symmetric $\mathrm{SL}(3, \mathrm{R})$ matrix.

We may conclude, therefore, that the dual action (2.31) is invariant under global SL(3,R) transformations. We now consider the relevant $\mathrm{SL}(2, \mathrm{R})$ subgroups that prove useful in generating non-trivial solutions with RR and NS-NS fields.

\section{B. SL $(2, R)_{\chi}$ symmetry}

The effective 4-D action (2.13) still exhibits the global SL(2, R) symmetry of the full 10-D action manifest in Eq. (2.5) 24,25. This becomes apparent by defining new scalar fields:

$$
\begin{aligned}
\frac{1}{2} \Phi \equiv u & \equiv \frac{1}{2} \varphi+\frac{\sqrt{3}}{2} y \\
v & \equiv \frac{\sqrt{3}}{2} \varphi-\frac{1}{2} y .
\end{aligned}
$$

The action given in Eq. (2.31) then takes the form

$$
\begin{array}{rl}
S_{4 *}=\int d^{4} & x \sqrt{-g}\left[R-\frac{1}{2}(\nabla u)^{2}-\frac{1}{2} e^{2 u}(\nabla \chi)^{2}-\frac{1}{2}(\nabla v)^{2}\right. \\
- & \left.\frac{1}{2} e^{\sqrt{3} v}\left(e^{-u}\left(\nabla \sigma_{2}\right)^{2}+e^{u}\left(\chi \nabla \sigma_{2}-\nabla \sigma_{1}\right)^{2}\right)\right] .
\end{array}
$$

The SL(3,R) matrix $U$ given in Eq. (3.4) can be written as

$$
U=\left(\begin{array}{cc}
e^{v / \sqrt{3}} M & -e^{v / \sqrt{3}} M \sigma \\
-e^{v / \sqrt{3}} \sigma^{T} M & e^{-2 v / \sqrt{3}}+e^{v / \sqrt{3}} \sigma^{T} M \sigma
\end{array}\right)
$$


where the symmetric $2 \times 2$ matrix $M$ is given in Eq. (2.6), and we have defined the vector

$$
\sigma \equiv\left(\begin{array}{c}
-\sigma_{1} \\
\sigma_{2}
\end{array}\right)
$$

This implies that Eq. (3.3) may be written as

$$
S_{4 *}=\int d^{4} x \sqrt{-g}\left[R+\frac{1}{4} \operatorname{Tr}\left[\nabla M \nabla M^{-1}\right]-\frac{1}{2}(\nabla v)^{2}-\frac{1}{2} e^{\sqrt{3} v} \nabla \sigma^{T} M \nabla \sigma\right] .
$$

The action remains invariant under the sub-group

$$
\bar{U}=\tilde{\Sigma}_{\chi} U \tilde{\Sigma}_{\chi},
$$

where

$$
\tilde{\Sigma}_{\chi}=\left(\begin{array}{cc}
\Sigma & 0 \\
0 & 1
\end{array}\right)
$$

and this corresponds to the $\mathrm{SL}(2, \mathrm{R})$ transformation

$$
\bar{M}=\Sigma M \Sigma^{T}, \quad \bar{g}_{\mu \nu}=g_{\mu \nu}, \quad \bar{\sigma}=\left(\Sigma^{T}\right)^{-1} \sigma, \quad \bar{v}=v .
$$

The transformation (3.13) acts non-linearly on the scalar fields $u$ and $\chi$ :

$$
\begin{array}{r}
e^{\bar{u}}=C^{2} e^{-u}+(D+C \chi)^{2} e^{u} \\
\bar{\chi} e^{\bar{u}}=A C e^{-u}+(B+A \chi)(D+C \chi) e^{u}
\end{array}
$$

and the pseudo-scalar axion fields transform as

$$
\begin{gathered}
\bar{\sigma}_{1}=A \sigma_{1}+B \sigma_{2} \\
\bar{\sigma}_{2}=C \sigma_{1}+D \sigma_{2} .
\end{gathered}
$$

When $C=-B=-1$ and $A=D=0$, Eq. (3.13) interchanges the two axion fields, $\sigma_{i}$, and inverts the 10-D string coupling $\bar{g}_{s}=\exp (\bar{\Phi})=g_{s}^{-1}=\exp (-\Phi)$ that is defined in terms of the $10-\mathrm{D}$ dilaton, $\Phi=2 u$. Thus, the strongly-coupled regime of the theory is mapped onto the weakly-coupled one, and vice-versa. The effective $4-\mathrm{D}$ dilaton field (2.14) transforms as $\bar{\varphi}=-\varphi / 2+\sqrt{3} v / 4$.

We refer to this as the $\mathrm{SL}(2, \mathrm{R})_{\chi}$ symmetry. It is the $\mathrm{SL}(2, \mathrm{R})$ symmetry of the $10-\mathrm{D}$ theory written in terms of the $4-\mathrm{D}$ variables 24.25]. The field $v$ determines the conformal factor (2.17) that relates the 4-D Einstein metric to the corresponding part of the 10-D Einstein metric (2.3). The invariance of both $v$ and the 4-D metric $g_{\mu \nu}$ implies that the corresponding components, $\hat{g}_{\mu \nu}=e^{\sqrt{3} v / 2} g_{\mu \nu}$, of the 10-D Einstein metric are also invariant. The radius of the internal space in the 10-D Einstein frame is $e^{-v / \sqrt{3}}$ and the complete 10-D Einstein metric (2.3) is therefore invariant, as in Eq. (2.9).

The effective interaction (2.41) may also be written in a $\mathrm{SL}(2, \mathrm{R})$ invariant form:

$$
V(u, v, \chi)=-\frac{1}{2} g^{\mu \nu} e^{-\sqrt{3} v} \mathcal{J}_{\mu}^{T} M \mathcal{J}_{\nu}
$$

where

$$
\mathcal{J}_{\mu}=\left(\begin{array}{c}
J_{\mu} \\
K_{\mu}
\end{array}\right)
$$

transforms as $\overline{\mathcal{J}}_{\mu}=\left(\Sigma^{T}\right)^{-1} \mathcal{J}_{\mu}$, and thus the covariantly conserved currents transform as

$$
\begin{aligned}
\bar{J}_{\mu} & =A J_{\mu}-B K_{\mu} \\
\bar{K}_{\mu} & =-C J_{\mu}+D K_{\mu} .
\end{aligned}
$$

This provides an interesting example of a non-trivial $(V \neq$ constant $) \operatorname{SL}(2, \mathrm{R})$ invariant interaction for the dilaton, despite the fact that there is no $\mathrm{SL}(2, \mathrm{R})$ invariant potential that can be derived from the dilaton alone [24]. The 
three-form field strengths can provide an effective interaction potential for the 10-D dilaton, $\Phi=2 u$, because they too transform under the $\mathrm{SL}(2, \mathrm{R})$ transformation.

The form of this interaction suggests that it may be possible to stabilise the 10-D dilaton due to interactions with the axion fields, as was recently noted in a similar context by Lukas, Ovrut and Waldram [31]. However, this is only possible if some other mechanism (in addition to the fields considered in this paper) operates to stabilise $v$ (the size of the compact space in the 10-D Einstein frame) and prevents the prefactor $e^{-\sqrt{3} v} \rightarrow 0$ in Eq. (3.18).

Finally, we note that although a general $\mathrm{SL}(2, \mathrm{R})$ matrix of the form given in Eq. (2.10) has three independent real parameters, there is a two-dimensional sub-group,

$$
\Sigma_{0} \equiv\left(\begin{array}{cc}
A^{-1} & 0 \\
B & A
\end{array}\right)
$$

which leaves the Lagrangian (2.31) invariant term by term. These transformations correspond either to a constant shift or rescaling of the axion fields, such that the three four-vectors, $e^{-\varphi} K_{\mu}, e^{(-\varphi+\sqrt{3} y) / 2}\left(J_{\mu}+\chi K_{\mu}\right)$ and $e^{(-\varphi-\sqrt{3} y) / 2}\left(L_{\mu}-\right.$ $\sigma_{2} K_{\mu}$ ) remain invariant. Thus the only non-trivial transformation is the "boost"

$$
\Sigma_{1} \equiv\left(\begin{array}{cc}
\cosh \theta & \sinh \theta \\
\sinh \theta & \cosh \theta
\end{array}\right)
$$

which introduces at most one new parameter $\theta$.

We can use the SL $(2, R)$ symmetry of the action to generate new four-dimensional solutions of the field equations. As an example in Section IV we will describe the homogeneous cosmological solutions with non-trivial $\chi$ field by applying the transformation given in Eq. (3.13) to the homogeneous dilaton-moduli-vacuum solutions.

\section{C. $\mathbf{Z}_{2}$ and $\mathrm{SL}(2, \mathbf{R})_{\sigma_{2}}$ symmetry}

The importance of the dual action is that a further SL $(2, \mathrm{R})$ symmetry may be uncovered. The NS-NS sector of the reduced action (2.13) is invariant under the 'T-duality' transformation $\bar{y}=-y$, corresponding to an inversion of the internal space. This $\mathrm{Z}_{2}$ symmetry can be extended to the $\mathrm{RR}$ sector of the theory and the dual action (2.31) is symmetric under the discrete transformation

$$
\bar{y}=-y, \quad \bar{\chi}=\sigma_{2}, \quad \bar{\sigma}_{2}=\chi, \quad \bar{\sigma}_{1}=-\sigma_{1}+\chi \sigma_{2},
$$

where the 4-D dilaton, $\varphi$, and 4-D Einstein frame metric remain invariant. Note, however, that because the volume of the internal space changes, the 10-D Einstein frame metric (related to the 4-D Einstein frame metric by the conformal factor given in Eq. (2.17)) is not invariant under this transformation. In terms of the conserved axion currents, defined in Eqs. (2.36), (2.35) and (2.43), the reflection symmetry (3.24) corresponds to

$$
\bar{y}=-y, \quad \bar{K}_{\mu}=-K_{\mu}, \quad \bar{J}_{\mu}=L_{\mu}, \quad \bar{L}_{\mu}=J_{\mu} .
$$

This reflection symmetry implies the existence of an alternative SL $(2, \mathrm{R})$ symmetry in the dual action which can be obtained from a combination of the $\mathrm{SL}(2, \mathrm{R})_{\chi}$ transformation given in Eq. (3.13) plus the reflection symmetry in Eq. (3.24). Analogously to Eqs. (3.5) and (3.6), but with $y \rightarrow-y$, we introduce the new scalar fields:

$$
\begin{aligned}
w & \equiv \frac{1}{2} \varphi-\frac{\sqrt{3}}{2} y \\
x & \equiv \frac{\sqrt{3}}{2} \varphi+\frac{1}{2} y .
\end{aligned}
$$

The dual effective action, Eq. (2.31), then takes the form

$$
\begin{array}{r}
S_{4 *}=\int d^{4} x \sqrt{-g}\left[R-\frac{1}{2}(\nabla w)^{2}-\frac{1}{2} e^{2 w}\left(\nabla \sigma_{2}\right)^{2}-\frac{1}{2}(\nabla x)^{2}\right. \\
\left.-\frac{1}{2} e^{\sqrt{3} x}\left(e^{w}\left(\nabla \sigma_{1}-\chi \nabla \sigma_{2}\right)^{2}+e^{-w}(\nabla \chi)^{2}\right)\right] .
\end{array}
$$

Defining the symmetric $2 \times 2$ matrix: 


$$
P \equiv\left(\begin{array}{cc}
e^{w} & \sigma_{2} e^{w} \\
\sigma_{2} e^{w} & e^{-w}+\sigma_{2}^{2} e^{w}
\end{array}\right)
$$

and the vector:

$$
\tau \equiv\left(\begin{array}{c}
\sigma_{1}-\chi \sigma_{2} \\
\chi
\end{array}\right)
$$

allows us to express this action as

$$
S_{4 *}=\int d^{4} x \sqrt{-g}\left[R+\frac{1}{4} \operatorname{Tr}\left[\nabla P \nabla P^{-1}\right]-\frac{1}{2}(\nabla x)^{2}-\frac{1}{2} e^{\sqrt{3} x} \nabla \tau^{T} P \nabla \tau\right] .
$$

This is manifestly invariant under the $\mathrm{SL}(2, \mathrm{R})$ transformation

$$
\bar{P}=\Sigma^{\prime} P \Sigma^{\prime T}, \quad \bar{g}_{\mu \nu}=g_{\mu \nu}, \quad \bar{\tau}=\left(\Sigma^{\prime T}\right)^{-1} \tau \quad \bar{x}=x
$$

and this implies that

$$
\begin{aligned}
e^{\bar{w}} & =C^{\prime 2} e^{-w}+\left(D^{\prime}+C^{\prime} \sigma_{2}\right)^{2} e^{w} \\
\overline{\sigma_{2}} e^{\bar{w}} & =\left(B^{\prime}+A^{\prime} \sigma_{2}\right)\left(D^{\prime}+C^{\prime} \sigma_{2}\right) e^{w}+A^{\prime} C^{\prime} e^{-w} \\
\bar{\chi} & =-C^{\prime}\left(\sigma_{1}-\sigma_{2} \chi\right)+D^{\prime} \chi \\
\bar{\sigma}_{1}-\bar{\chi} \bar{\sigma}_{2} & =A^{\prime}\left(\sigma_{1}-\sigma_{2} \chi\right)-B^{\prime} \chi .
\end{aligned}
$$

We refer to this as the $\mathrm{SL}(2, \mathrm{R})_{\sigma_{2}}$ symmetry of the action. It should be emphasised that this is not the $10-\mathrm{D}$ $\mathrm{SL}(2, \mathrm{R})_{\chi}$ symmetry recast in terms of the $4-\mathrm{D}$ action. The $\mathrm{SL}(2, \mathrm{R})_{\sigma_{2}}$ transformation mixes the $\sigma_{2}$ axion field with $w$. This latter field is not the $10-\mathrm{D}$ dilaton, because it includes an additional contribution from the modulus field $y$. Thus, the radius of the internal dimensions transforms non-trivially and the $10-\mathrm{D}$ Einstein metric is not invariant under (3.32).

Comparison of Eq. (3.5) with Eq. (3.26) and Eq. (3.6) with Eq. (3.27) implies that the discrete transformation $y \leftrightarrow-y$ is equivalent to $u \leftrightarrow w$ and $v \leftrightarrow x$. Moreover, Eqs. 2.6) and (3.29) imply that the reflection symmetry (3.24) is equivalent to $M \leftrightarrow P$. Thus, the $\mathrm{SL}(2, \mathrm{R})_{\sigma_{2}}$ symmetry transformation is formally equivalent to the $\mathrm{Z}_{2}$ transformation (3.24), followed by the $\mathrm{SL}(2, \mathrm{R})_{\chi}$ transformation (3.13), followed by another $\mathrm{Z}_{2}$ transformation $(3.24$ ).

Finally, we recall that we were able to employ the conserved currents for $\sigma_{i}$ to provide reduced equations of motion for $\varphi, y$ and $\chi$ in Eqs. (2.38 2.40), where the effective interaction potential Eq. (3.18) is invariant under the SL(2,R) $\chi$ transformation. The reflection symmetry (3.24) implies that analogous reduced field equations for $\varphi, y$ and $\sigma_{2}$ may be derived by employing the conserved currents for $\sigma_{1}$ and $\chi$. Substituting the definitions of $K_{\mu}$ and $L_{\mu}$, Eqs. (2.35) and (2.43), into the equations of motion implies that

$$
\begin{aligned}
\square w & =e^{2 w}\left(\nabla \sigma_{2}\right)^{2}-\frac{\partial W}{\partial \varphi} \\
\square x & =-\frac{\partial W}{\partial y} \\
\nabla^{\mu}\left(e^{2 w} \nabla_{\mu} \sigma_{2}\right) & =-\frac{\partial W}{\partial \chi},
\end{aligned}
$$

where the effective interaction potential $W$ between the fields $\varphi, y$ and $\sigma_{2}$ is given by

$$
W\left(w, x, \sigma_{2}\right)=-\frac{1}{2} e^{\sqrt{3} x} \nabla \tau^{T} P \nabla \tau=-\frac{1}{2} g^{\mu \nu} e^{-\sqrt{3} x} \mathcal{L}_{\mu}^{T} P \mathcal{L}_{\nu}
$$

and

$$
\mathcal{L}_{\mu} \equiv\left(\begin{array}{c}
L_{\mu} \\
-K_{\mu}
\end{array}\right)
$$

$\mathcal{L}_{\mu}$ transforms as $\overline{\mathcal{L}}_{\mu}=\left(\Sigma^{\prime T}\right)^{-1} L_{\mu}$ under the $\mathrm{SL}(2, \mathrm{R})_{\sigma_{2}}$ transformation in Eq. (3.32) and the conserved currents therefore transform as

$$
\begin{aligned}
\bar{L}_{\mu} & =A^{\prime} L_{\mu}+B^{\prime} K_{\mu} \\
\bar{K}_{\mu} & =C^{\prime} L_{\mu}+D^{\prime} K_{\mu}
\end{aligned}
$$




\section{FOUR-DIMENSIONAL HOMOGENEOUS COSMOLOGIES}

The symmetry transformations described in Section III may be employed to generate solutions with non-trivial NS-NS and RR form fields from any given solution to the dilaton-moduli-vacuum field equations. 4-D cosmological solutions are of particular interest as they might describe the early evolution of our own universe. Homogeneous and isotropic Friedmann-Robertson-Walker (FRW) solutions were presented in [17]. In this Section we extend the analysis to spatially homogeneous but anisotropic Bianchi universes. These models admit a three-dimensional Lie group of isometries, $G_{3}$, acting on the surfaces of homogeneity. The space-like surfaces have a metric, $h_{a b}=h_{a b}(t)$ $(a, b=1,2,3)$, where $t$ represents cosmic time and the four-dimensional line element in the string frame is given by

$$
d s^{2}=-d t^{2}+h_{a b} \omega^{a} \omega^{b}
$$

where $\omega^{a}$ are the one-forms for each Bianchi type. The three--metric may be diagonalized:

$$
h_{a b}(t)=e^{2 \alpha(t)}\left(e^{2 \beta(t)}\right)_{a b}
$$

where

$$
\beta_{a b}=\operatorname{diag}\left[-2 \beta_{+}, \beta_{+}-\sqrt{3} \beta_{-}, \beta_{+}+\sqrt{3} \beta_{-}\right]
$$

is a traceless matrix that determines the anisotropy in the models and $e^{\alpha}$ is the 'averaged' scale factor. Under the conformal transformation given in Eq. (2.15), the averaged scale factor in the Einstein frame is given by $\tilde{\alpha}=\alpha-\varphi / 2$, but the definition of the variables $\beta_{ \pm}$is conformally invariant.

The antisymmetric structure constants, $C^{a}{ }_{b c}$, of the Lie algebra of $G_{3}$ may be written as $C^{a}{ }_{b c}=m^{a d} \epsilon_{d b c}+\delta^{a}{ }_{[b} a_{c]}$, where $m^{a b}=m^{b a}, a_{c} \equiv C^{a}{ }_{a c}, \epsilon_{a b c}$ is a covariantly constant three-form and indices are raised and lowered with $h^{a b}$ and $h_{a b}$, respectively. The Jacobi identity then implies that $m^{a b} a_{b}=0$ and the Lie algebra is in the Bianchi class A or B if $a_{b}=0$ or $a_{b} \neq 0$, respectively [32]. The Lagrangian formulation of the class B models is ambiguous because the condition $a_{b} \neq 0$ leads to non-vanishing spatial divergence terms. Such problems do not arise in the class A, however, and this includes the Bianchi types $\mathrm{I}, \mathrm{II}, \mathrm{VI}_{0}, \mathrm{VII}_{0}, \mathrm{VIII}$ and IX. We therefore restrict our discussion to these models?.

A Lagrangian for the Bianchi class A models may be derived from the dual action (2.31) by integrating over the spatial variables. If the scalar fields are themselves homogeneous on the spatial hypersurfaces, it follows that the action can be written as

$$
S_{4 *}=\int d T\left[L_{g}+L_{m}\right]
$$

where

$$
\begin{aligned}
L_{g} & \equiv-6 \dot{\tilde{\alpha}}^{2}+6 \dot{\beta}_{+}^{2}+6 \dot{\beta}_{-}^{2}+{ }^{(3)} R\left(\tilde{\alpha}, \beta_{ \pm}\right) \\
L_{m} & \equiv \frac{1}{2} \dot{\varphi}^{2}+\frac{1}{2} \dot{y}^{2}+\frac{1}{2} e^{\sqrt{3} y+\varphi} \dot{\chi}^{2}+\frac{1}{2} e^{-\sqrt{3} y+\varphi} \dot{\sigma}_{2}^{2}+\frac{1}{2} e^{2 \varphi}\left(\dot{\sigma}_{1}-\chi \dot{\sigma}_{2}\right)^{2}
\end{aligned}
$$

represent the gravitational and matter Lagrangians, respectively, a dot denotes differentiation with respect to the time coordinate in the Taub gauge [34]

$$
T \equiv \int d \tilde{t} e^{-3 \tilde{\alpha}}=\int d t e^{-3 \alpha-\varphi}
$$

and the scalar curvature of the three-surfaces is given by ${ }^{(3)} R=-\left(m_{a b} m^{a b}-\left(m_{a}^{a}\right)^{2} / 2\right)$.

It follows, therefore, that the action for each specific Bianchi type is uniquely determined once the functional form of the three--curvature has been specified. The advantage of employing the time variable (4.7) is that the gravitational and matter sectors of the action (2.31) are effectively decoupled from one another. This implies that the vacuum solutions $\left(\dot{\chi}=\dot{\sigma}_{i}=0\right)$ for the dilaton and moduli fields are given linearly by

\footnotetext{
${ }^{2}$ It should be remarked, however, that the analysis is also valid for Bianchi types III and V and the Kantowski-Sachs model. See, e.g., Ref. [33] for a full discussion of the Bianchi types.
} 


$$
\begin{aligned}
& \varphi=\varphi_{*}+\left(\sqrt{2 E} \cos \xi_{*}\right) T \\
& y=y_{*}+\left(\sqrt{2 E} \sin \xi_{*}\right) T
\end{aligned}
$$

for all Bianchi types, where $E$ is an arbitrary positive constant of integration which represents the kinetic energy associated with Lagrangian $L_{m}$. Consequently, these solutions correspond to straight-line trajectories in the $(\varphi, y)$ field space. They are tilted at an arbitrary angle $\xi_{*}$ to the $\varphi$-axis. Note that the averaged scale factor in the Einstein frame, $e^{\tilde{\alpha}}$, is the solution for a homogeneous cosmology with a stiff perfect fluid equation of state [35]. For instance, in the case of the spatially flat, Bianchi type I model, we have $\tilde{\alpha} \propto T$, and the scale factor in the string frame is then given by $e^{\alpha}=e^{\tilde{\alpha}+(\varphi / 2)}$.

\section{A. Single form field}

An $\mathrm{SL}(2, \mathrm{R})$ transformation given by Eq. (3.13) or (3.32) generates the general solution with a single, nontrivial form field $\chi$ or $\sigma_{2}$, respectively, when applied to the general homogeneous dilaton-moduli-vacuum solution [13, 17, 36, 37, 16, 14]. If initially $\chi=\sigma_{1}=\sigma_{2}=0$, then the $\mathrm{SL}(2, \mathrm{R})_{\chi}$ transformation (3.13) generates a single timelike, covariantly conserved four-current $L_{\mu}$, and therefore a conserved charge $L=e^{\varphi+\sqrt{3} y} \dot{\chi}=2|C D| \cos \left(\xi_{*}-\pi / 3\right)$. This places a lower limit on the variable $u$, defined in Eq. (3.5), and thus the 10-D dilaton, $\Phi$. On the other hand, the $\mathrm{SL}(2, \mathrm{R})_{\sigma_{2}}$ transformation (3.32) generates a conserved charge $J=e^{\varphi-\sqrt{3} y} \dot{\sigma}_{2}=2\left|C^{\prime} D^{\prime}\right| \cos \left(\xi_{*}+\pi / 3\right)$, placing a lower limit on the variable $w$ defined in Eq. (3.26).

Since the matter component (4.6) of the dual action has the same form for all Bianchi types, we may conclude that the trajectories of the corresponding solutions in the $(\varphi, y)$ plane are identical to those for the isotropic FRW solutions [17. All solutions with a single excited form field exhibit a single bounce between asymptotic dilaton-moduli-vacuum solutions. The coupling of the form field to the dilaton and moduli determines the reflection angle of the bounce [17].

\section{B. Two form fields}

The field equations imply that the only consistent solution with one trivial and two non-trivial form fields corresponds to the case where the field strength of the NS-NS field vanishes, $H^{(1)}=0$. Thus, the pseudo-scalar axion field $\sigma_{1}$ may be eliminated by employing the definition (2.26), which implies that $\dot{\sigma}_{1}=\chi \dot{\sigma_{2}}$. It then follows that the two non-trivial form fields become decoupled. Thus, their field equations may be directly integrated to yield $e^{\varphi+\sqrt{3} y} \dot{\chi}=L$ and $e^{\varphi-\sqrt{3} y} \dot{\sigma}_{2}=J$, as in the case of only a single non-trivial form field described above.

The field equations for the dilaton and moduli fields may be written in the form of the SU(3) Toda system [38]. Defining the new variables

$$
q_{ \pm} \equiv \varphi \pm \frac{y}{\sqrt{3}}
$$

the matter Lagrangian given in Eq. (4.6) becomes

$$
L_{m}=\frac{1}{8}\left(\dot{q}_{+}+\dot{q}_{-}\right)^{2}+\frac{3}{8}\left(\dot{q}_{+}-\dot{q}_{-}\right)^{2}-V\left(q_{ \pm}\right),
$$

where

$$
V\left(q_{ \pm}\right) \equiv \frac{1}{2}\left(J^{2} e^{q_{+}-2 q_{-}}+L^{2} e^{q_{-}-2 q_{+}}\right)
$$

represents an effective potential. This implies that the field equations take the form

$$
\begin{aligned}
& \ddot{q}_{-}=J^{2} e^{q_{+}-2 q_{-}} \\
& \ddot{q}_{+}=L^{2} e^{q_{-}-2 q_{+}}
\end{aligned}
$$

subject to the constraint equation

$$
\frac{1}{8}\left(\dot{q}_{+}+\dot{q}_{-}\right)^{2}+\frac{3}{8}\left(\dot{q}_{+}-\dot{q}_{-}\right)^{2}+V=E,
$$


where $E$ is a positive constant.

The general solution to Eqs. (4.13)-(4.15) is of the form 114,36, 37, 17

$$
e^{q_{-}}=\sum_{i=1}^{3} A_{i} e^{-\lambda_{i} T}, \quad e^{q_{+}}=\sum_{i=1}^{3} B_{i} e^{\lambda_{i} T}
$$

where $\sum_{i} \lambda_{i}=0$. This implies that $\lambda_{\min }<0$ and $\lambda_{\max }>0$ and the asymptotic solution for $\varphi$ and $y$ as $T \rightarrow-\infty$ is therefore given by

$$
e^{\varphi} \sim e^{-\left(\lambda_{\max }-\lambda_{\min }\right) T / 2}, \quad e^{y} \sim e^{\sqrt{3}\left(\lambda_{\max }+\lambda_{\min }\right) T / 2}
$$

On the other hand, we have

$$
e^{\varphi} \sim e^{\left(\lambda_{\max }-\lambda_{\min }\right) T / 2}, \quad e^{y} \sim e^{\sqrt{3}\left(\lambda_{\max }+\lambda_{\min }\right) T / 2}
$$

in the limit $T \rightarrow+\infty$.

The asymptotic solutions (4.17) and (4.18) correspond to straight lines in the $(\varphi, y)$ plane. This is similar behaviour to that of the single form field solutions discussed above. More specifically, the incoming trajectories arrive from infinity $(\varphi \rightarrow \infty)$ at an angle $\xi_{*}$ to the $\varphi$-axis and are reflected back at an angle $-\xi_{*}$. The constraint equation (4.15) implies the upper bound, $V \leq E$, on the potential (4.12) and this restricts the range of $\xi_{*}$ to $\left|\xi_{*}\right| \leq \pi / 6[17$.

\section{Three form fields}

We wish to investigate here the general cosmological solution with all three form fields non-trivial [17. The general FRW solutions to the type IIB string action presented in Eq. (2.31) can be obtained from the dilaton-vacuum solutions by a general $\mathrm{SL}(3, \mathrm{R})$ transformation. This is equivalent to three (non-commuting) $\mathrm{SL}(2, \mathrm{R})$ transformations: $\mathrm{SL}(2, \mathrm{R})_{\chi} \times \mathrm{SL}(2, \mathrm{R})_{\sigma_{2}} \times \mathrm{SL}(2, \mathrm{R})_{\chi}$ which yields a complicated analytic form. The general solution exhibits a sequence of bounces between asymptotic vacuum states. We find that the time-dependence of the fields $\chi$ and $\sigma_{2}$ induces lower bounds on the variables $u$ and $w$, respectively, as seen in the single form-field solutions. In the general solution this results in a lower bound on $\varphi=u+w$.

The general type IIB solution contains a non-vanishing NS-NS form-field, but can always be obtained from the Toda system of Eq. (4.11) where $H^{(1)}=0$ by a single $\mathrm{SL}(2, \mathrm{R})$ transformation (3.13). The asymptotic behaviour of $\varphi$ and $y$ is invariant under this transformation. This follows since $u \rightarrow \infty$ asymptotically for all solutions in the Toda system 3 and, from Eq. (3.14), we obtain $\bar{u} \rightarrow u$ in the general solution. We also have $\bar{v}=v$ and thus $\varphi$ and $y$ are invariant in this limit. Thus, trajectories in $(\varphi, y)$ field-space come in at an angle $\xi_{*}$ and leave at an angle $-\xi_{*}$, where $-\pi / 6 \leq \xi_{*} \leq \pi / 6$

\section{DISCUSSION}

In this paper we have considered a 4-D effective action derived by compactifying the 10-D type IIB supergravity action on an isotropic six-torus. Only the variations of the form fields on the external 4-D spacetime were considered and, although many degrees of freedom present in the full 10-D theory were omitted, the interactions between the form fields of the NS-NS and RR sectors were included. These interactions are such that a dual action, Eq. (2.31), may be derived, where the field strengths of the two-forms are interchanged with the one-form field strengths of two pseudo-scalar axion fields. The five scalar fields in this action parametrize an $\mathrm{SL}(3, \mathrm{R}) / \mathrm{SO}(3)$ coset.

The global symmetry of the $10-\mathrm{D}$ effective action, $\mathrm{SL}(2, \mathrm{R})_{\chi}$, is respected by the dual action. However, this action is also invariant under a discrete $\mathrm{Z}_{2}$ reflection symmetry and this implies the existence of a second, global $\mathrm{SL}(2, \mathrm{R})$ symmetry, $\mathrm{SL}(2, \mathrm{R})_{\sigma_{2}}$, corresponding to the mirror image of the original $\mathrm{SL}(2, \mathrm{R})_{\chi}$ symmetry. This results in an effective interaction potential for the dilaton, modulus and $\mathrm{RR}$ axion fields that may be expressed in a $\mathrm{SL}(2, \mathrm{R})$ invariant form. The $\mathrm{SL}(2, \mathrm{R})_{\chi}$ symmetry mixes the $\mathrm{RR}$ axion and $10-\mathrm{D}$ dilaton and leaves the internal space in the

\footnotetext{
${ }^{3}$ An exceptional case is when $u \rightarrow u_{*}$ asymptotically, where $u_{*}$ is a constant. In this case $\bar{u} \rightarrow$ constant, though not necessarily $u_{*}$, but the qualitative behaviour is the same.
} 
10-D Einstein frame invariant. Thus, both the 10-D and 4-D Einstein frame metrics transform as singlets under this symmetry. However, the $\mathrm{Z}_{2}$ symmetry is a 'T-duality' which inverts the size of the internal space. Although the 4-D Einstein metric remains invariant, the $10-\mathrm{D}$ Einstein metric is not invariant under the $\mathrm{Z}_{2}$ (or the resulting $\left.\mathrm{SL}(2, \mathrm{R})_{\sigma_{2}}\right)$ symmetry.

It is interesting to compare the symmetries of the dual action (2.31) with the more familiar symmetries of the NS-NS sector of the string effective action. It is well known that there is an $\mathrm{SL}(2, \mathrm{R})$ symmetry between the dilaton and the NS-NS axion when the RR form-fields are zero 12. The NS-NS three-form $H_{\mu \nu \lambda}^{(1)}$ is dual to $e^{2 \varphi} \nabla_{\kappa} \sigma_{1}$ in this case and Eq. (2.31) can be written as

$$
S_{4 *}=\int d^{4} x \sqrt{-g}\left[R-\frac{1}{2}(\nabla y)^{2}+\frac{1}{4} \operatorname{Tr}\left(\nabla N \nabla N^{-1}\right)\right]
$$

where

$$
N \equiv\left(\begin{array}{cc}
e^{\varphi} & \sigma_{1} e^{\varphi} \\
\sigma_{1} e^{\varphi} & e^{-\varphi}+\sigma_{1}^{2} e^{\varphi}
\end{array}\right)
$$

is a member of the group $\mathrm{SL}(2, \mathrm{R})$. Thus, the NS-NS sector is invariant under a global $\mathrm{SL}(2, \mathrm{R})$ transformation 12]

$$
\bar{N}=\Sigma N \Sigma^{T}, \quad \bar{g}_{\mu \nu}=g_{\mu \nu}, \quad \bar{y}=y
$$

and there is also an independent $\mathrm{Z}_{2}$ symmetry:

$$
\bar{y}=-y .
$$

The $\mathrm{SL}(2, \mathrm{R})$ symmetry (5.3) is not respected by the terms involving the RR field strengths, but there is a striking similarity between the symmetry of the complete dual action (2.31) and the restricted NS-NS action (5.1). In the case of the NS-NS sector alone, the $\mathrm{SL}(2, \mathrm{R})$ and $\mathrm{Z}_{2}$ symmetries act on different fields and thus commute, whereas this is not so in the type IIB effective action (2.31). In the former case, the $\mathrm{SL}(2, \mathrm{R})$ symmetry is its own mirror image under the $\mathrm{Z}_{2}$ reflection, but when the $\mathrm{RR}$ fields are included, we find that the mirror image of the original $\mathrm{SL}(2, \mathrm{R})_{\chi}$ symmetry is a second $\mathrm{SL}(2, \mathrm{R})_{\sigma_{2}}$ symmetry. Both actions include an $\mathrm{SL}(2, \mathrm{Z}) \mathrm{S}$-duality and a discrete $\mathrm{Z}_{2} \mathrm{~T}$-duality, and we have seen that many qualitative features of the NS-NS solutions alone [13] are shared by the more general type IIB solutions with both NS-NS and RR fields included [17].

The action (2.31) corresponds to a truncation of the bosonic sector of $N=8$ supergravity. Although the U-duality group of this theory is known to be the full $\mathrm{E}_{7(7)}$ group, the advantage of employing the truncated action is that exact solutions to the field equations may be derived. We have demonstrated in Section IV that these symmetries of the dual action are powerful tools for generating new 4-D backgrounds with non-trivial RR fields from vacuum solutions or solutions where only the NS-NS sector is non-trivial. The cosmological solutions at early or late times approach the dilaton-moduli-vacuum solutions asymptotically where the NS-NS and RR form fields become fixed. However the presence of the form-fields restricts the allowed range of the asymptotic solutions. It is interesting to note that the pre-Big Bang scenario proposed by Gasperini and Veneziano [39] is based on solutions to the low-energy string effective action that emerge from the weakly-coupled dilaton-moduli-vacuum. These solutions are not typical of the general axion-dilaton-moduli solutions where the form fields place a lower bound on the dilaton coupling. Even if the form fields are set to zero then we expect quantum fluctuations with spectra that can be computed assuming the Bunch-Davies vacuum state in the short-wavelength limit. The SL $(2, \mathrm{R})$ symmetry of the NS-NS sector previously has been used to obtain the perturbation spectra of the axion and dilaton fields 115,16] and a similar technique using the $\mathrm{SL}(3, \mathrm{R})$ symmetry could be applied to include perturbations in the RR sector.

More generally, the symmetries discussed in this paper may be employed in conjuction with previous analyses of non-compact symmetries to generate a wide class of anisotropic and inhomogeneous cosmologies or membrane solutions from a given vacuum or NS-NS solution [18, 19, 40 42]. A solution containing excited dilaton and axion fields may be found by applying an $\mathrm{O}(d, d)$ transformation to an appropriate vacuum solution of Einstein gravity. This is relevant to the study of inhomogeneous string cosmologies where the homogeneity is broken along one direction. Such models are known as Einstein-Rosen solutions and admit two abelian isometries and, therefore, a global $\mathrm{O}(2,2)$ symmetry [43,44]. Beginning with a vacuum Einstein-Rosen solution, an $\mathrm{O}(2,2)$ transformation, followed by the $\mathrm{SL}(2, \mathrm{R})$ transformation (5.3), would lead to a general class of inhomogeneous NS-NS cosmologies. These solutions would then serve as the starting point for generating the RR sector via the symmetries discussed in Section III. A similar procedure may be employed to generate plane wave solutions involving RR fields [45]. Solutions of this nature are interesting because they model the collision of gravitational waves in the very early universe [46]. They would therefore allow the effects of stringy excitations on the propagation of tensor perturbations to be investigated. 


\section{ACKNOWLEDGEMENTS}

EJC and JEL are supported by the Particle Physics and Astronomy Research Council (PPARC), UK. We thank J. Maharana for useful discussions.

[1] A. Sen, Int. J. Mod. Phys. A9, 3707 (1994);

E. Alvarez, L. Alvarez-Gaume, and Y. Lozano, Nucl. Phys. Proc. Suppl. B41, 1 (1995);

E. Witten, Nucl. Phys. B443, 85 (1995);

A. Sen, "Unification of String Dualities", hep-th/9609176;

J. Polchinski, Rev. Mod. Phys. 68, 1245 (1996);

M. Duff, Int. J. Mod. Phys. A11, 5623 (1996);

P. S. Aspinwall, Nucl. Phys. Proc. Suppl. B46, 30 (1996);

J. H. Schwarz, Nucl. Phys. Proc. Suppl. B55, 1 (1997);

C. Vafa, "Lectures on Strings and Dualities", hep-th/9702201.

[2] A. Giveon, M. Porrati, and E. Rabinovici, Phys. Rep. 244, 77 (1994).

[3] C. Hull and P. Townsend, Nucl. Phys. B438, 109 (1995).

[4] C. M. Hull, Nucl. Phys. B468, 113 (1996).

[5] C. Vafa, Nucl. Phys. B469, 403 (1996).

[6] J. Dai, R. G. Leigh, and J. Polchinski, Mod. Phys. Lett. A4, 2073 (1989);

M. Dine, P. Huet, and N. Seiberg, Nucl. Phys. B322, 301 (1989).

[7] E. Cremmer and B. Julia, Phys. Lett. 80B, 48 (1978); Nucl. Phys. B159, 141 (1979).

[8] K. A. Meissner and G. Veneziano, Phys. Lett. B267, 33 (1991).

[9] J. Maharana and J. Schwarz, Nucl. Phys. B390, 3 (1993).

[10] M. Gasperini, J. Maharana, and G. Veneziano, Phys. Lett. B296, 51 (1992).

[11] M. Gasperini, J. Maharana, and G. Veneziano, Phys. Lett. B272, 277 (1991).

[12] A. Shapere, S. Trivedi, and F. Wilczek, Mod. Phys. Lett. A6, 2677 (1991);

A. Sen, Mod. Phys. Lett. A8, 2023 (1993).

[13] E. J. Copeland, A. Lahiri, and D. Wands, Phys. Rev. D 50, 4868 (1994).

[14] N. Kaloper, Phys. Rev. D 55, 3394 (1997).

[15] E. J. Copeland, R. Easther, and D. Wands, Phys. Rev. D 56, 874 (1997).

[16] E. J. Copeland, J. E. Lidsey, and D. Wands, Nucl. Phys. B506, 407 (1997).

[17] E. J. Copeland, J. E. Lidsey, and D. Wands, Phys. Rev. D 57, 625 (1998).

[18] R. Poppe and S. Schwager, Phys. Lett. B393, 51 (1997).

[19] A. Feinstein, R. Lazkoz, and M. A. Vazquez-Mozo, Phys. Rev. D 56, 5166 (1997).

[20] J. H. Schwarz, Nucl. Phys. B226, 269 (1984);

P. Howe and P. C. West, Nucl. Phys. B238, 181 (1984).

[21] N. Marcus and J. H. Schwarz, Phys. Lett. 115B, 111 (1982).

[22] E. Bergshoeff, C. Hull, and T. Ortin, Nucl. Phys. B451, 547 (1995).

[23] J. H. Schwarz, Phys. Lett. B360, 13 (1995); ibid B364, 252 (1995); E. Bergshoeff and H. J. Boonstra, Phys. Rev. D 53, 7206 (1996).

[24] J. Maharana, Phys. Lett. B402, 64 (1997).

[25] S. Roy, "On the S-duality of toroidally compactified type IIB string effective action", hep-th/9705016.

[26] E. Bergshoeff, H. J. Boonstra, and T. Ortin, Phys. Rev. D 53, 7206 (1996).

[27] A. Andrianopoli, R. D'Auria, S. Ferrara, P. Fre, R. Minasian, and M. Trigiante, Nucl. Phys. B493, 249 (1997).

[28] E. J. Copeland, A. Lahiri, and D. Wands, Phys. Rev. D 51, 1569 (1995).

[29] D. V. Gal'tsov, A. A. Garcia, and O. V. Kechkin, Class. Quantum Grav. 12, 2887 (1995).

[30] D. Maison, Gen. Rel. Grav. 10, 717 (1979).

[31] A. Lukas, B. A. Ovrut, and D. Waldram, Nucl. Phys. B509, 169 (1998).

[32] G. F. R. Ellis and M. A. H. MacCallum, Commun. Math. Phys. 12, 108 (1969).

[33] J. Wainwright and G. F. R. Ellis, Dynamical Systems in Cosmology (Cambridge University Press, Cambridge, England, 1997).

[34] A. H. Taub, Ann. Math. 53, 472 (1951).

[35] J. P. Mimoso and D. Wands, Phys. Rev D52, 5612 (1995).

[36] A. Lukas, B. A. Ovrut, and D. Waldram, Phys. Lett. B393, 65 (1997); Nucl. Phys. B495, 365 (1997);

A. Lukas and B. A. Ovrut, "U-duality covariant M-theory cosmology", hep-th/9709030. 
[37] H. Lü, S. Mukherji, and C. N. Pope, Phys. Rev. D 55, 7926 (1997);

H. Lü, J. Maharana, S. Mukherji, and C. N. Pope, ibid 57, 2219 (1998).

[38] B. Kostant, Adv. Math. 34, 195 (1979).

[39] M. Gasperini and G. Veneziano, Astropart. Phys. 1, 317 (1993).

[40] N. A. Batakis and A. A. Kehagias, Nucl. Phys. B449, 248 (1995);

N. A. Batakis, Phys. Lett. B353, 39 (1995); Phys. Lett. B353, 450 (1995);

N. A. Batakis and A. A. Kehagias, Phys. Lett. B356, 223 (1995);

J. D. Barrow and M. P. Dabrowski, Phys. Rev. D 55, 630 (1997).

[41] A. Strominger, Nucl. Phys. B343, 167 (1990);

E. Bergshoeff, H. J. Boonstra, and T. Ortin, Phys. Rev. D 53, 7206 (1996).

[42] J. D. Barrow and K. E. Kunze, Phys. Rev. D 55, 623 (1997).

[43] E. Verdager, Phys. Rep. 229, 1 (1993).

[44] I. Bakas, Nucl. Phys. B428, 374 (1994).

[45] S. Kar, A. Kumar, and G. Sengupta, Phys. Lett. B375, 121 (1996).

[46] J. B. Griffiths, Colliding Plane Waves in General Relativity (Clarendon Press, Oxford, 1991). 